DOE/ER/61015--Tl T/

DE92 013654

\title{
STATISTICAL EXAMINATION OF CLIMATOLOGICAL DATA RELEVANT TO GLOBAL TEMPERATURE VARIATION
}

Progress Report

July 1991 - January 1992

H.L. Gray, Richard F. Gunst, and Wayne A. Woodward

Department of Statistical Science

Southern Methodist University

Dallas, Texas 75275

January 1992

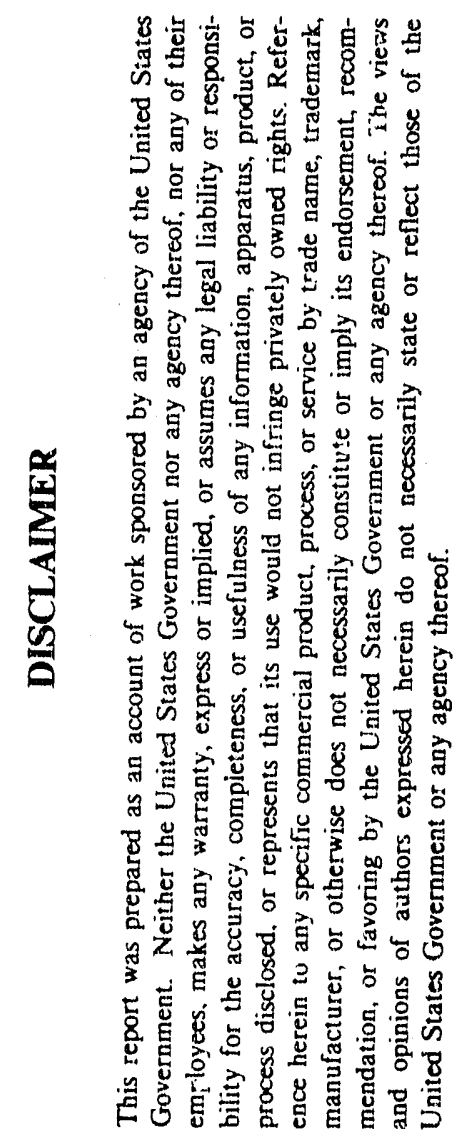

PREPARED FOR THE U.S. DEPARTMENT OF ENERGY UNDER GRANT NUMBER DE-FG05-90ER61015

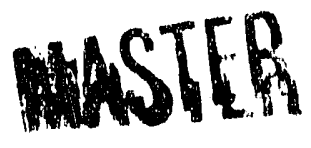




\section{INTRODUCTION}

The research group at Southern Methodist University has been involved in the examination of climatclogical data as specified in the proposal. Our efforts have resulted in three papers which have been submitted to scholarly journals, as well as several other projects which should be completed either during the next six months or sext year. In the following, we discuss our results to date along with projected progress within the next six months.

\section{COMPLETED REPORTS}

\section{Testing for Trend in the Global Temperature Data}

One of the key projects with which we have been involved is testing for the existence of a trend in the global temperature data. In recent years a number of statistical tests have been proposed for testing the hypothesis that global warming is occurring. The standard approach is to examine one or two of the more prominent global temperature data sets by letting $Y_{t}=a+b t+E_{t}$, where $Y_{t}$ represents the temperature at time $t$ and $E_{t}$ represents error from the trend line, and testing the hypothesis that $b=0$. Several authors (e.g. Bloomfield and Nychka, 1991; Bloomfield, 1991; Kuo, Lindberg, and Thomson, 1990) have applied these tests for trend to determine whether or not a significant trend exists in the widely referenced global temperature data sets, and have generally concluded that there is a significant trend in the data of the form which should be incorporated into forecasts.

However, certain autoregressive-moving average (ARMA) models are also very reasonable models for these data due to the random trends present in their realizations. In particular an ARMA model is a model of the form

$$
\left(1-\phi_{1} B-\cdots-\phi_{p} B^{p}\right)\left(Y_{t}-\mu\right)=\left(1-\theta_{1} B-\cdots-\theta_{q} B^{q}\right) a_{t}
$$

where $a_{t}$ is white (uncorrelated) noise, the $\phi_{j}$ 's and $\theta_{j}$ 's are real constants, and $B^{k} Y_{t}=$ $Y_{t-k}$. The values $p$ and $q$ are called the model orders. The model specifies that the 
current value of the process at time $t$, i.e $Y_{t}$, is a linear combination of the previous $p$ values of the process plus a linear combination of noise components. In our research, we propose ARMA models for the warming trend data sets. It is shown that the correlation structure for these models is not nearly strong enough for the ARMA-based forecasts to predict any continued increase. However, when data were generated from these ARMA models, we demonstrated that the tests for trend quite often incorrectly predict a trend to continue, i.e. the short term random trends in these realizations cause the tests to erroneously conclude $b \neq 0$ (and thus treat them as if they were long-term trends). Of course, the projections that the warming trend will extend into the future are largely based on such factors as the buildup of atmospheric greenhouse gases, and we hasten to add that we do not conclude that there is or is not a warming trend. We simply make the point that these statistical tests for testing $H_{O}: b=0$ have little or no ability to distinguish between realizations from ARMA models with a high correlation between successive values and those from models of the form $Y_{t}=a+b t+E_{t}$. Our conclusion is that based solely on the available temperature deviation series, it is difficult to conclude that these temperature data suggest that the trend will continue over any extended length of time. We further conclude that trend tests based on the model $Y_{t}=\mu_{t}+E_{t}$ for the purpose of prediction or inference concerning future behavior should be used with caution.

To further emphasize thess points, we considered the yearly average Zurich sunspot numbers for the 108 years sparined by the Hansen and Lebedeff temperature series, i.e. 1880-1987 (see Waldineder, 1961 and NOAA, 1990). These data have a pseudoperiodic behavior with a period of about 11 years along with a general tendency for the amplitudes of the oscillations to increase over this time span. We applied the tests for trend to this series and noted that these testa concluded that there is a significant linear trend in the sunspot data for the past 108 years and would hence forecast a continued trend in sunspots. This is of course a finding which would not be viewed as reasonable by the scientific community, and we would not believe forecasts that indicated that the increasing trend observed over the last 108 years will continue over any extended period of time. On the contrary, since we have a long history of sunspot data we know that the behavior we have observed over the last 108 years is simply a part of the natural cycle of sunspot behavior. In particular, for the 239 years 
from 1749-1987, there have been several periods of increasing and of decreasing amplitudes. It should now be clear why we have advised that it is at best questionable to conclude, if the aforementioned tests reject $\mathrm{H}_{\mathrm{O}}: b=0$, that this implies there is a "forecastable" trend.

The results of this investigation have been written up and submitted to the Journal of Climate for possible publication. A copy of the manuscript has also been sent to Mike Riches. A shortened version of the paper will appear as a DOE Research Summary.

\section{Defining and Estimating Mean Global Temperature Change}

Mean global temperature change is a concept whose definition usually must be inferred by how it is estimated. Explicitly lacking in all the major articles on global warming is any definition of a fixed (theoretical) quantity referred to as "mean global temperature change." Without such a definition, the assessment of estimation methodologies relies on the "reasonableness" of the respective approaches, an assessment that is very subjective. On the other hand, a definition of a theoretical quantity such as mean global temperature change permits a rigorous evaluation of estimation strategies, evaluations that can be either theoretical or empirical.

At any fixed time $t$, each location $x$ on the earth has an associated temperature $T_{t}(\boldsymbol{x})$. Current estirnation procedures focus on temperature deviations or "anomalies" $a_{t}(x)$, where $a_{t}(x)=T_{t}(x)-\tau(x)$ is the temperature deviation at time $t$ for location $x$, and $\tau(x)$ represents the temperature mean for location $x$ over a stated period of time. The definition of mean global temperature change used in our research is

$$
\delta_{t}=\int_{R} a_{t}(x) w(x) d x / \int_{R} w(x) d x,
$$

where the region $R$ in this definition can represent any geographic area of interest, including the entire earth. If the region $R$ is defined by a discrete set of locations $\left\{x_{i}\right\}$, the integrals can be approximated by summations :

$$
\delta_{t}=\sum_{i} a_{t}\left(x_{i}\right) w\left(x_{i}\right) A_{i} / \sum_{i} w\left(x_{i}\right) A_{i}
$$


where the subscripts $i$ denote a partitioning of the region $R$ and $A_{i}$ is the area of the $i$ th subregion in the partitioning.

Based on the above integral definition of a mean global temperature anomaly, commonly used distance weighting of anomalies, i.e. linear distance weighting used by Hansen and Lebedeff (1987) and inverse distance weighting used by Jones, et al. (1986a, 1986b, 1986c) and Jones (1988), are shown to be inferior to ordinary (uniform weighting) and robust (m-estimatcr weighting) averages for the estimation of regional means. On the other hand, gridding (typically applied by researchers in this area without any justification in the specific context of estimating regional temperature anomaly means) is shown to improve the performance of all the averages studied when station locations are not evenly dispersed throughout a region, the usual situation in practice. Data reuse in the context of gridding, such as that employed by Hansen and Lebedeff (1987), is shown to often lead to more serious biases than those resulting from the choice of a less than optimal estimator.

As an application of the above findings, we estimated mean global temperature anomalies for the years 1880-1988 based on the NCAR monthly station data and using m-estimator weighting, gridding, and no station reuse. The m-estimator weighting has the advantage that isolated errors in station data do not have a severe effect on the results. It should be noted, however, that the results obtained with uniform weighting and m-estimator weighting were very similar. Our results suggest that temperature anomalies in the late 1800's might not be as small as heretofore reported. Thus, comparisons of the early part of the record with more recent temperature anomalies do not show as large a change as is commonly reported. This difference is not so great that previous conclusions made from the temperature records are negated; however, they clearly demonstrate the need for the research that is being conducted. Moreover, these findings leave open the question of whether further investigations will suggest additional modifications of the estimates.

This paper has been submitted for publication to Journal of Climate. A copy of the paper has also been sent to Mike Riches. 


\section{The Effect of Initial Conditions on Autcregressive Models for Global Temperature} Data

A common approach to studying the impact of greenhouse gases on climate is via cornputer simulation using general circulation models (GCMs) which integrate current understanding of the various factors influencing climate through a comprehensive mathematical model. There is much current debate over the degree of validity that can be attributed to the output from a GCM since our understanding of the input factors which effect climate is far from perfect. In a recent article, Tsonis (1991) examines the sensitivity of GCMs to initial conditions. As stated by Tsonis, an obvious way to determine the effect of the initial conditions on predictions oitained from a GCM is to obtain a control realization (i.e. a sample over time), modify the initial conditions, obtain another realization, and compare the results. Since this could be excessively time consuming, Tsonis suggests modeling the global temperature series of Jones, et al. (1986a, 1986b, 1986c) and Jones (1988) by fitting a fourth order autoregressive process, $\mathrm{AR}(4)$ (i.e. a model as in (1) with $p=4$ and $q=0$ ), to the data and then using such a model to generate additional realizations with modified starting values. He assumes that the sensitivity of GCMs to initial conditions is similar to that of the AR model, and he then reasons that if slightly changing the initial values in simulated realizations from the $A R(4)$ model results in very dissimilar realizations, this provides evidence that the GCMs themselves are too sensitive to initial conditions to trust predictions made from simulations.

Our results show that $T$ sonis is incorrect when he concludes that predictions from a stationary autoregressive model fitted to temperature data are saxisitive to the starting values; on the contrary, the opposite is true. It is well known that for any stationary AR process, if the sample size is large relative to the order of the process, the initial values will have little effect on forecasts. We show that different realizations with the same starting values from the AR(4) model fit by Tsonis typically show little similarity beyond 30 years and literally no resemblance (in the sense of correlation) beyond 50 years. The "different" realizations indicated here ha "e different and independently derived noise sequences. Tsonis repeatedly generated different realizations in this manner from processes with slightly different starting values and attributed the dissimilarity after 30 and 50 years to the difference in the starting values. In fact, 
however, this dissimilarity is not due to initial values, but instead it is due to the correlation structure and noise sequence.

To make this point another way, we generated two samples with different starting values but with the same noise sequence and correlation structure. This corresponds to the same set of random conditions driving the weather, but from different starting values, and thus provides a clearer picture of the sensitivity to the starting values themselves. For a given stationary AR model it is the noise sequence and the correlation structure between observations that will determine the eventual behavior of the realization, and we demonstrate that realizations from this AR(4) model (and hence with the same correlation function) based on the same noise sequence but different starting values typically merge within 30 years, again illustrating their insensitivity to the starting values.

Thus, we have been able to show that the claim of Tsonis that the stationary autoregressive model fit to the global temperature data is overly sensitive to starting values is incorrect. The question still remains open, however, as to the sensitivity of the GCMs themselves to initial conditions.

The results of this investigation have been submitted to EOS and a copy of the full report has been sent to Mike Riches.

\section{PROJECTS IN PROGRESS}

\section{Analysis of Sunspot Data Using GARMA Models}

For data such as the mean global temperature deviations, $\mathrm{CO}_{2}$ data, or 'ther data related to climate, the correlation structure between observations may persist over long intervals of time. In this case, it is often true that neither a signal + noise model nor an ARMA model are appropriate. A possible solution to this dilemma is the Gegenbauer ARMA (GARMA) model which is a long memory model introduced by Gray, Zhang, and Woodward (1989). This model is an extension of the ARMA model which allows for long term dependency in stationary models, i.e. allows for stationary 
models with slowly damping autocorrelations. The GARMA model is given by

$$
\left(1-\phi_{1} B-\cdots-\phi_{p} B^{p}\right)\left(1-2 u B+B^{2}\right)^{\lambda}\left(\mathrm{Y}_{t}-\mu\right)=\left(1-\theta_{1} B-\cdots-\theta_{q} B^{q}\right) a_{t}
$$

where $u$ specifies the frequency at which the long memory behavior occurs and $\lambda$ essentially indicates how slowly the autocorrelations damp. The GARMA model appears to be particularly appropriate for data related to climate change, and we will investigate the validity of this observation by examining forecast models obtained for a number of different climate related data sets.

One such analysis involves the sunspot data, and we will use traditional ARMA models as well as GARMA models. In the original proposal we discussed a preliminary AR.MA and GARMA analysis of the sunspot data. Cynthia Kalkomey (1991), a recent Ph.D. student under H.L. Gray, has extended the results shown there for forecasting sunspot data using both ARMA and GARMA models, and her results indicate that forecasts from the GARMA models are superior to those obtained using ARMA techniques. We are planning to write a report which involves modeling and forecasting yearly sunspot averages using the most current sunspot series. A report will be written describing the results by Gray, Kalkomey, and Woodward.

\section{Careful Modeling of the Temperature and $\mathrm{CO}_{2}$ Dato.}

We are preparing a paper which will give details of the modeling procedures which resulted in the ARMA models for the temperature data sets. These models were given by Woodward and Gray (1991 - referenced in Appendix) without details of the modeling involved. The temperature data series given by Gunst, et al. (1991 referenced in Appendix) will also be modeled and compared with the models for the Hanser and Lebedeff and the Jones, et al. series. GARMA models for these series will also be considered. Appropriate ARMA and GARMA models will also be found and compared for the atmospheric $\mathrm{CO}_{2}$ measured at Mauna Loa (Keeling et al., 1989). A manuscript will be written concerning this research by Gray and Woodward. 


\section{The Effects of Changing Station Coverage}

The global temperature estimation of Gunst, Basu, and Brunell (1991 - referenced in Appendix) does not address the issue of changing staticn coverage and its effect on the temperature series. A preliminary survey of the relevant literature reveals two somewhat contrasting positions. The urban heat island effect, partially due to changes in station coverage, is cited as a serious concern; yet changes in station coverage is generally regarded as having a small effect on mean global temperature anomaly calculations. Investigations are planned to determine what, if any, effects occurred as a result of the change from Smithsonian to Weather Bureau records in the 1960 's. Also, the temperature series will be examined which use only stations for which long records exist in order to avoid the problems of changing coverage. The report of these investigations will be written by Gunst, Basu and Brunell.

\section{Transfer Function Modeling}

Techniques are being developed at SMU for better identification of transfer function models, i.e. for models in which the prediction of future values of a process $Y_{t}$ depend not only on present and past values of that series but also on another exogenous series $X_{t}$ In our application, we will examine the joint behavior of $\mathrm{CO}_{2}$, temperature, sunspots and possibly other covariates. While this work is currently in progress, most likely the results will be forthcoming during the third year of the project. More details about the work in this area will be given in the proposal for the third year. This research is being done by Woodward, Gray, and Cheng (a graduate student).

\section{Kriging Approach to Estimating Mean Global Temperature}

Kriging is a method for analyzing spatial data, and in our case has applications to the estimation of mean global temperature from siation data. This technique depends on a covariance structure which is essentially a spatial counterpart to the autocovariance structure important in the analysis of time series. Critical components in the fitting of kriging models are the assumptions about the form of the special covariance matrix and the procedures used to fit the assumed matrix. This work is currently in progress but results will likely not be available until the third year. More details concerning this research will be given in the proposal for the third year. This 
work is being done by Gunst, Brunell, and Hong (a graduate student).

\section{Estimating Temperatures Using Tree Ring Data}

A real need in the area of climate change research is for usable sequences of "temperature" data which extend backward beyond the last 130 years or so for which thermometer-based readings are available. Other data sets such as the $\mathrm{O}^{18}$ readings found in ocean sediments provide indications of temperature in past climates. However, these series are usually on a long time scale with readings typically being approximately 2000 years apart. This time scale provides very little information concerning the types of natural climate variability which have occurred over 100 year periods in the past. In an effort to assess whether temperature variation over the last 100 years is consistent with patterns since ca. 1600 and as a means of testing the predicative capacity of climate models, we have initiated a project using dendroclimatic data.

Dendroclimatology provides a method of obtaining temperature and precipitation reconstructions up to the last 10,000 years. We have focused on a series of data sets that contain reconstructions for the last three hundred years. Most of these are from the Northern Hemisphere (e.g. Fritts, 1991). The first step in this analysis is to assess the validity of the temperature reconstructions. Using the NCAR data we have selected sites where there is measured monthly temperature available and compared these data with temperature reconstructions from the same region. Using sites where there is a high degree of correlation between measured and reconstructed temperaiure, the next step in our analysis is to begin to statistically evaluate the reconstructed tersperatures on both a site and regional basis.

Certain preliminary results from this investigation should be forthcoming this spring, and if the area of research shows promise we anticipate this research carrying over into the third year. This research is primarily being performed by Hietala, Hill, and Wendorf from the Department of Anthroplogy at Southern Methodist University. 


\section{REFERENCES}

Bloomfield, Peter (1991), "Trends in Global Temperature," unpublished manuscript.

Bloomfield, P. and Nychka, D. (1991). "Climate Spectra and Detecting Climate Change," unpublished manuscript.

Fritts, H.C. (1991). Reconstructing Large-Scale Climatic Patterns from Tree-Ring Data: A Diagnostic Analysis. University of Arizona Press.

Gray, H.L., Zhang, N. and Woodward, W.A. (1989). "On Generalized Fractional Processes," Journal of Time Series Analysis 10, 233-257.

Hansen, J. and Lebedeff, S. (1987). "Global Trends of Measured Surface Air Temperature," Journal of Geophysical Research 92, 13345-13372.

Hill, C. and Wendorf, F. (1991). "Paleoclimatic Dynamics in the Eastern Sahara (North Africa) Based on Late Quaternary Sediments Containing Acheulian and Middle Paleolithic Artifacts," Geological Society of America Abstracts with Programs 23(5), 356.

Jones, P.D., S.C.B. Raper, R.S. Bradley, H.F. Diaz, P.M. Kelly, and T.M.L. Wigley, (1986a), "Northern Hemisphere Surface Air Temperature Variations: 1851-1984," J. Clim. Appl. Meterol., 25, 161-179.

Jones, P.D., S.C.B. Raper, and T.M.L. Wigley (1986b), "Southern Hemisphere Surface Air Temperature Variations: 1851-1984.," J. Clim. Appl. Meterol., 25, 1213-1230.

Jones, P.D., T.M.L. Wigley, and P.B. Wright, (1986c), "Global Temperature Variations Between 1861 and 1984," Nature 322, 430-434.

Jones, P.D., (1988), "Hemispheric Surface Air Temperature Variations: Recent Trends and an Update to 1987," Journal of Climate 1, 654-660. 
Jones, P.D. and Wigley, T.L. (1990). "Global Warming Trends," Scientific American, August, 84-91.

Kalkomey, C. (1991). Modeling Quasi-Periodic and Seasonal Long Memory Processes. Ph.D. Dissertation, Department of Statistical Science, Southern Methodist University, Dallas, Texas.

Keeling, C.D., Bacastow, R.B., Carter, A.F., Piper, S.C., and Whorf, T.P. (1989). American. Geophysical Union, Geophysics Monograph 55, 165-236.

Kuo, C., Lindberg, C. and Thomson, D.J. (1990), "Coherence Established Between Atmospheric Carbon Dioxide and Global Temperature," Nature 343, 709-714.

NOAA (1990), "Solar-Geophysical Data Prompt Reports," February.

Tsonis, A.A. (1991), "Sensitivity of the Global Climate System to Initial Conditions," EOS 72, 313.

Waldmeier, M. (1961), The Sunspot Activity in the Years 1610-1960. Zurich: Zurich Schulthess and Co. 


\section{APPENDIX}

The following manuscripts have been written under the support of this grant and have been submitted for review to technical journals during the first six months of this grant year.

(1) Woodward, W.A. and $G_{1}$ y, H.L. (1991), "Global Warming' and the Problem of Testing for Trend in Time Series Data," submitted to Journal of Climate.

(2) Gunst, R.F., Basu, S., and Brunell, R. (1991), "Defining and Estimating Mean Global Temperature Change," submitted to Journal of Climate.

(3) Gray, H.L. and Woodward, W.A. (1991), "The Effect of Initial Conditions on Autoregressive Models for Global Temperature Data," submitted to EOS. 

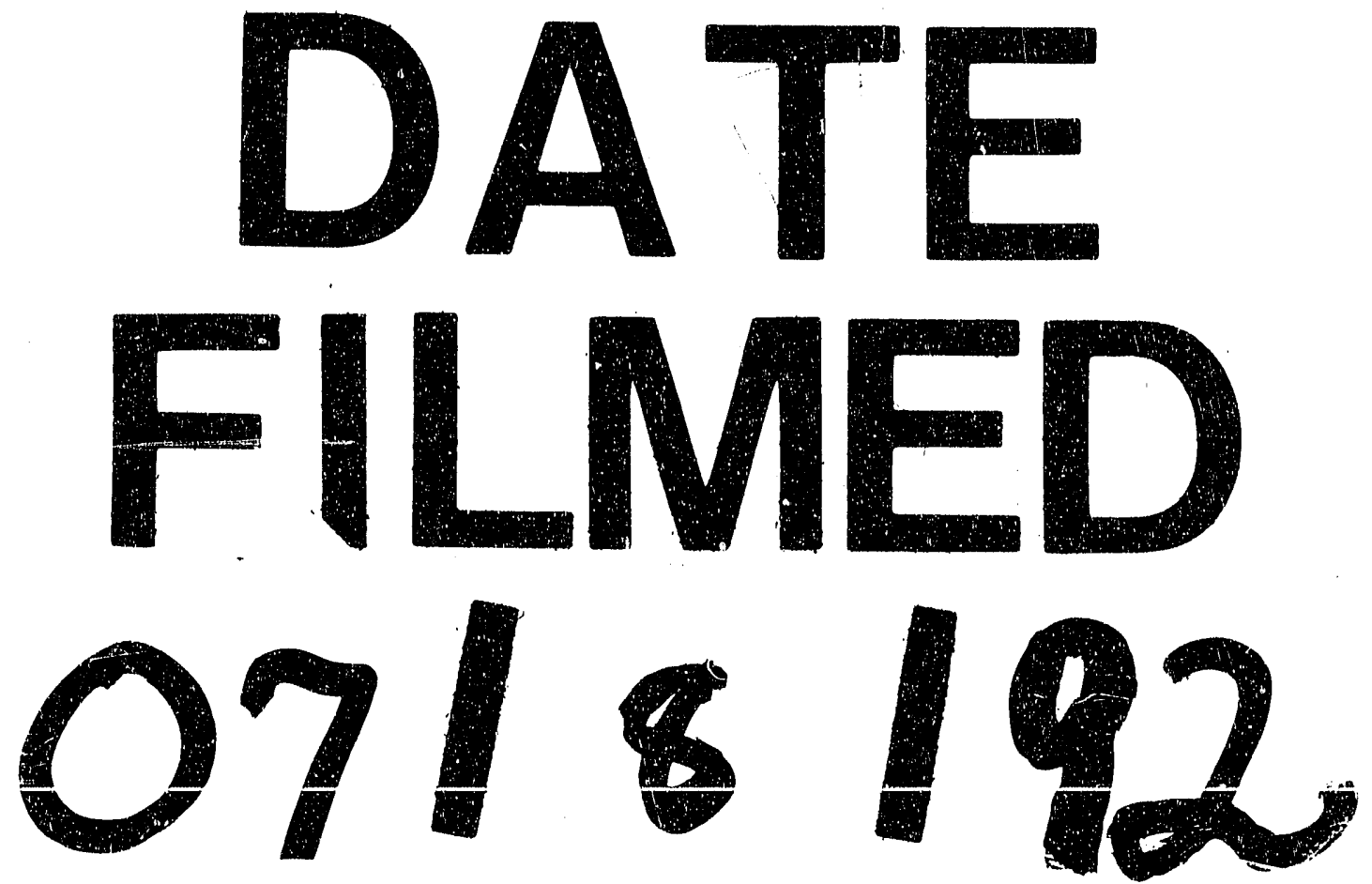
